\title{
Organização Espacial, Saúde e Qualidade de Vida: Análise Espacial e Uso de Indicadores na Avaliação de Situações de Saúde*
}

\author{
Spatial Organization, Health and Quality of Life: Use of \\ Spatial Analysis and Indicators in Health Situation Analysis
}

Christovam de Castro Barcellos

Departamento de Informações em Saúde/FIOCRUZ

Paulo Chagastelles Sabroza

Escola Nacional de Saúde Pública/FIOCRUZ

Paulo Peiter

Departamento de Geografia/UFRJ

Luisa Iñiguez Rojas

Centro de Estudios de Salud y Bienestar Humano/Universidad de La Habana

\begin{abstract}
Resumo
O espaço é uma categoria de síntese e convergência onde se expressam os diversos processos envolvidos nas condições de vida, ambiente e saúde das populações. Ao longo desses processos, os resultados obtidos pela análise de indicadores são sensíveis à seleção desses indicadores, das fontes de informação, da escala de análise, de unidades espaciais de referência e dos métodos de análise espacial. O objetivo deste trabalho é levantar o papel da análise espacial na avaliação das situações de saúde, que engloba a descrição de perfis epidemiológicos, de qualidade de vida e de condições ambientais. Conhecer a estrutura e a dinâmica espacial da população é o primeiro passo para a caracterização de situações de saúde. Além disso, permite o planejamento de ações de controle e alocação de recursos. A análise espacial propicia o restabelecimento do contexto no qual um evento de saúde ocorre, contribuindo para o entendimento dos processos socioambientais envolvidos.
\end{abstract}

\section{Palavras-Chave}

Análise Espacial; Ambiente; Análise de Situação de Saúde; Indicadores de Saúde.

\section{Summary}

Space is a synthetic and convergent category in which the processes involved in the quality of life, environment and health conditions of populations are expressed. Through these processes, the results obtained by the analysis of indicators are sensitive to the choice of indicators, information sources, scale of analysis, spatial reference units and methods of spatial analysis. The objective of this work is to define the role of spatial analysis in the health situation assessment, which includes the description of the epidemiological profiles, quality of life and environmental conditions. The first step for characterizing the health situation is to describe the structure and spatial dynamics of the population. Spatial analysis permits the planning of control actions and resource allocation as it allows to describe and understand the social and enviromental processes in which health events occur.

Key Words

Spatial Analysis; Environment; Health Situation Analysis; Health Indicators.

\footnotetext{
* Trabalho apresentado ao I Seminário Nacional Saúde e Meio Ambiente como contribuição para a discussão do tema.

Endereço para correspondência: Av Brasil, 4.365 - Manguinhos - Rio de Janeiro/RJ. CEP: 21.041-230.

E-mail: xris@cict.fiocruz.br

Informe Epidemiológico do SUS 2002; 11(3) : 129 - 138
} 


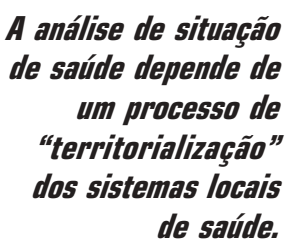

Informe Epidemiológico do SUS

\section{Introdução}

Se a doença é uma manifestação do indivíduo, a situação de saúde é uma manifestação do lugar. Os lugares, dentro de uma cidade ou região, são resultado de uma acumulação de situações históricas, ambientais e sociais que promovem condições particulares para a produção de doenças. Uma das questões importantes para o diagnóstico de situações de saúde, nesse sentido, é o desenvolvimento de indicadores capazes de detectar e refletir condições de risco à saúde advindos de condições ambientais e sociais adversas. Esses indicadores devem permitir a identificação dos lugares, suas relações com a região, bem como a relação entre a população e seu território. É nessas relações que se desenvolvem meios propícios para o desenvolvimento de doenças e também para seu controle.

A análise de situações de saúde corresponde a uma vertente da vigilância da saúde que prioriza a análise da saúde de grupos populacionais definidos em função de suas condições de vida. Depende, portanto, de um processo de "territorialização" dos sistemas locais de saúde, de modo a reconhecer porções do território segundo a lógica das relações entre condições de vida, saúde e acesso aos serviços de saúde. ${ }^{1} \mathrm{~A}$ análise de situação de saúde tem uma lógica territorial, porque no espaço se distribuem populações humanas segundo similaridades culturais e socioeconômicas. O Estado organiza-se e implementa ações nessa base territorial, que é, por isso, palco de conflitos e também da gestão de setores como a saúde. ${ }^{2} \mathrm{~A}$ análise de fenômenos de saúde no espaço serve antes de tudo para a síntese de indicadores epidemiológicos, ambientais e sociais. Essa análise pode ser realizada, utilizando-se diversas escalas e unidades espaciais de referência. Os diferentes recortes a que pode ser submetido o espaço separam estruturas espaciais e grupos populacionais segundo critérios que podem realçar ou suavizar diferenças entre valores, ou mesmo gerar flutuações aleatórias desses indicadores. ${ }^{3}$
Este texto é resultado de discussões realizadas para a preparação do I Seminário Nacional de Saúde e Ambiente no Processo de Desenvolvimento. Durante esse encontro, em julho de 2000, foi realizada a oficina sobre organização espacial, saúde e ambiente. A principal questão que se pretende encaminhar neste trabalho é: como a análise espacial pode auxiliar na avaliação das situações de saúde, que engloba tanto a descrição de perfis epidemiológicos, de condições ambientais e de qualidade de vida? Para isso, é discutida a construção e análise de indicadores, bem como os diferentes usos que o espaço vem adquirindo nas análises de saúde e suas implicações teórico-metodológicas.

\section{O papel do espaço nas análises de situação de saúde}

Uma das noções mais elementares que se vem difundindo sobre o espaço é similar ao conceito utilizado na matemática. Em um plano multidimensional, vazio a priori, onde se desenvolvem funções, é possível colocar pontos, linhas e superfícies. O desenvolvimento de técnicas de estatística espacial e de análise de redes tem incentivado esse tipo de abordagem espacial para estudos em saúde. Esse espaço-geometria é contínuo e representado por um conjunto de relações entre objetos num plano cartesiano e tem como premissa que os elementos espaciais próximos (contíguos ou ligados por conexões) compartilham condições socioambientais semelhantes.

Um dos exemplos de abordagem estatística na saúde e ambiente é o levantamento de doenças próximas a fontes conhecidas de poluição. ${ }^{4} \mathrm{~A}$ distância dos eventos em relação à fonte suspeita de contaminação é utilizada como parâmetro estimativo da exposição humana a agentes de risco. Essa estimativa é particularmente útil nos estudos em que se deseja avaliar doses a que foram submetidos grupos humanos em longos períodos de tempo. ${ }^{5}$ A modelagem estatística espacial é também utilizada para o estudo de difusão de doenças ou agentes infecciosos. Nesse 
caso, os dados de saúde são separados em períodos de tempo, de modo que permita analisar ou simular sequiencialmente quadros evolutivos das epidemias. ${ }^{6}$

Uma segunda concepção do espaço admite a existência de um mosaico de subespaços com características fisiográficas e humanas próprias, não necessariamente semelhantes aos vizinhos. $\mathrm{O}$ espaço-região tanto pode ser concebido como uma realidade concreta, construída ao longo de sua história, como ser objeto de criação intelectual a partir de parâmetros estatísticos que a caracterizem em função de objetivos preestabelecidos. ${ }^{7}$ Essa abordagem tem como principais pressupostos a homogeneidade interna e a independência entre unidades espaciais de agregação e análise de dados. ${ }^{8} 9$ Esse enfoque é principalmente utilizado nos chamados "estudos ecológicos", tendo como unidade de análise uma área geográfica, uma região.

Uma terceira idéia sobre espaço procura entendê-lo como um conjunto de lugares, resultado da interação singular de grupos sociais com seu entorno mais próximo, ${ }^{10}$ ou a coexistência de "grupos socioespaciais particulares" que, apesar de compartilhar o mesmo espaço, possuem diferentes modos de vida, relações de trabalho e relações com o ambiente. ${ }^{11}$ Essa forma de entender o espaço como espaço-lugar permite tanto o estudo quanto o planejamento de ações diferenciadas sobre grupos socioespaciais dentro da mesma região. Na Região Amazônica, por exemplo, índios e garimpeiros possuem modos de vida relacionados à sua inserção social que podem produzir perfis epidemiológicos divergentes. Os lugares seriam, na concepção de Milton Santos, ${ }^{12}$ palco da vida cotidiana e mesmo uma resistência ao processo de globalização e homogeneização do espaço. Segundo essa abordagem, de influência humanística, o espaço, ao invés de uma mera localização, deve ser substituído pelo lugar como experiência, com padrão de troca e significados próprios. ${ }^{13}$ São exemplos dessa abordagem alguns estudos etno- gráficos sobre a malária na Amazônia, e outras endemias no Brasil e na África. ${ }^{14}$

Cada uma dessas abordagens possui limitações específicas, relacionadas aos conceitos e métodos utilizados. A utilização da categoria espaço na análise de situações de saúde não se limita à mera localização de eventos de saúde, isso porque o lugar atribui a cada elemento constituinte do espaço um valor particular. ${ }^{15}$ As potencialidades e limitações do uso do espaço nessas análises decorrem da diversidade de seus próprios conceitos e conteúdos.

O espaço é, ao mesmo tempo, produto e produtor de diferenciações sociais e ambientais, processo que tem importantes reflexos sobre a saúde dos grupos sociais envolvidos. Esse processo envolve o valor do solo e o uso do solo, de modo a valorizar regiões com melhores condições ambientais e desvalorizar áreas degradadas. Desse modo, uma cidade "produz" o lugar dos ricos, dos pobres e da indústria, bem como estabelece fluxos de circulação de bens e serviços. Uma cidade é necessariamente heterogênea, resultado da permanente ação da sociedade sobre a natureza. Por outro lado, esse espaço produzido socialmente exerce pressões econômicas e políticas sobre essa sociedade, criando condições diferenciadas para sua utilização por grupos sociais. Lugares sujeitos a exteriorizações negativas próximos a indústrias poluentes, com baixa oferta de serviços urbanos - tendem a concentrar moradores de baixa renda em busca de empregos ou locais de moradia mais baratos. ${ }^{16}$ Dessa forma, o espaço "acumula" as transformações ocorridas, num processo permanente de renovação, refletindo mais seu passado do que propriamente o presente.

\section{Recortes do espaço e medição de desigualdades}

O estabelecimento de escalas de trabalho, como empregadas na cartografia, é reconhecidamente artificial. O espaço geográfico é constituído por um sistema de objetos e ações com inúmeras articulações verticais e horizontais. ${ }^{12}$ 
Nesse espaço, manifestam-se variáveis globais de ação local e outros processos de origem local com pequena amplitude e resultados também locais. A organização de redes no espaço permite, cada vez mais, que esses processos sejam simultâneos e abrangentes.

A necessidade de se controlar a extensão do estudo impõe uma escala de trabalho, definida a partir da escolha de unidades espaciais de referência. Para que sejam dispostos em mapas, cada um dos dados, disponíveis nos diversos sistemas de informação em saúde, devem ser referenciados a uma unidade de análise geográfica. As unidades básicas de referência geográfica desses dados têm sido, por imposição desses sistemas, os diversos níveis da administração pública, como o município ou o estado. Os processos tanto ambientais quanto sociais, que promovem ou restringem situações de risco à saúde, não estão limitados a essas fronteiras administrativas. Outras formas de estratificação e visualização de indicadores em mapas devem ser buscadas. Entre as possíveis unidades espaciais de agregação de dados ambientais e sanitários, encontram-se o setor censitário, o bairro, a bacia hidrográfica, o distrito sanitário, o distrito administrativo e o município. ${ }^{17}$

Com o uso de Sistemas de Informações Geográficas (SIG), a implantação de endereços nos registros de saúde e o uso crescente de Sistemas de Posicionamento Global (GPS) nas ações de vigilância em saúde, é possível dispor estes eventos de saúde na forma de pontos em um mapa com escala local. ${ }^{18}$ A principal vantagem desta estratégia de georreferenciamento de dados é a possibilidade de serem produzidas diferentes formas de agregação de dados, construindo-se indicadores para diferentes unidades espaciais conforme o interesse. Um mesmo ponto (evento de saúde) pode estar contido em diferentes tipos de unidades espaciais: um bairro, uma bacia hidrográfica ou um distrito sanitário, por exemplo, definidos por polígonos nos mapas. Essa propriedade implica a adoção de um rigor geométrico que deve estar presente na fase de planejamento e de construção da base cartográfica. Para que haja uma relação unívoca entre ponto e polígono, as unidades espaciais devem cobrir toda a área de trabalho e uma área não pode ser coberta por mais de um polígono, isto é, não pode haver vazios ou sobreposição de unidades.

Outros critérios podem ser listados para a escolha de uma dessas unidades espaciais, o que influirá sobre a forma e estrutura que terão a base de dados e a base cartográfica. Entre esses critérios, destacam-se os seguintes: ${ }^{17}$

a) a presença e qualidade do registro dessas unidades nos bancos de dados;

b) o reconhecimento da unidade espacial por parte da população;

c) a disponibilidade de dados sobre saúde e ambiente na unidade;

d) a existência de grupos populacionais organizados e de instâncias administrativas do Estado na unidade; e

e) a máxima homogeneidade interna e heterogeneidade externa das unidades.

Essas exigências técnicas na manipulação, tanto de dados tabulares quanto de dados cartográficos, dificultam a adoção de critérios menos rígidos para os estudos espaciais, restringindo o conceito de espaço a um conjunto de unidades estanques, o espaço-região. A utilização de técnicas de análise de redes, interpolação e suavização de dados espaciais, permite dissolver os limites previamente estabelecidos entre unidades espaciais. Nesse caso, o conceito de espaço se aproxima ao de espaço-geometria. A adoção de limites difusos (fuzzy) para unidades espaciais, ideal para o estudo do lugar, é prejudicada pelas normas operacionais de sistemas de informação. ${ }^{9}$

A escala de trabalho se delineia com o estabelecimento a priori das unidades de agregação de dados e da extensão do território de trabalho. De cada uma dessas unidades emergem fatores próprios, 
específicos da escala de trabalho. Por exemplo, uma quadra é formada por um conjunto de residências. Outro conjunto maior de residências pode formar um bairro. O bairro, por sua vez, pode possuir uma representação política popular, uma associação de moradores ou que lhe caracterize. Essa organização política não existe em escalas maiores (as quadras) nem menores (distritos ou municípios). Os indicadores socioambientais referentes a uma determinada unidade espacial não são necessariamente formados pela agregação de dados do nível individual. Podem emergir da unidade espacial de análise considerada. ${ }^{19}$ Inclui-se, nesse caso, a maior parte das variáveis ambientais, que não se referem aos indivíduos, mas são intrínsecas à unidade espacial.

O desenvolvimento de estudos que envolvem a análise espacial deve aprimorar a capacidade de combinação entre diferentes escalas de análise, por meio de níveis de agregações sucessivos e continentes das unidades anteriores, bem como deve implementar variáveis de localização dos eventos de interesse nos sistemas de informação utilizados em saúde e ambiente.

\section{Desigualdades e diferenças de qualidade de vida}

Os reflexos da desigualdade social sobre as condições de saúde são objeto de estudo na área da saúde há várias décadas. Por intermédio dos chamados "estudos ecológicos", têm-se procurado correlações entre indicadores epidemiológicos e socioeconômicos, geralmente provenientes de censos. Pessoas e áreas de pior nível socioeconômico apresentam, quase invariavelmente, piores condições de saúde.

Os diversos indicadores setoriais de condições de vida estão, muitas vezes, relacionados no chamado "nível ecológico", de agregados espaciais: uma área com alto nível de renda tende a oferecer boa cobertura dos serviços de saneamento, equipamentos de saúde e de educação. ${ }^{20} \mathrm{Da}$ mesma maneira, áreas pobres, principalmente aquelas situadas em cinturões das grandes cidades do terceiro mundo, tendem a ser excluídas de todos esses serviços. Esses indicadores de condições de vida freqüentemente estão associados à densidade demográfica, isto é, à concentração de riqueza e de população. ${ }^{21}$ Nesse caso, o uso de índices sintéticos de qualidade de vida reforça a tendência de polarização do espaço em áreas ricas e pobres. Aquelas com serviços e investimentos por parte do Estado e que atraem uma população com maior poder de decisão, e outras, com menores recursos, nas quais se concentram grupos populacionais sem maiores possibilidades de escolha. O espaço, nesse caso, é concebido mais como produtor do que como produto de diferenciações sociais e epidemiológicas. As variáveis usualmente empregadas para a identificação de desigualdades entre condições de vida são históricas, não casuais, nem conjunturais. A mais elementar das variáveis geográficas, a densidade demográfica, pode ter importantes repercussões sobre a difusão de doenças, principalmente das transmissíveis. Essa variável é resultado da capacidade humana de, por meio da divisão territorial do trabalho, produzir excedentes e tecnologia e organizar estruturas de poder.

A estratégia de estratificação de grupos populacionais por meio da fragmentação do espaço em regiões ou "áreas homogêneas" pode ajudar a identificar subespaços de condições de vida semelhantes. ${ }^{20}$ Essa abordagem espacial não substitui, no entanto, os estudos de estratificação da população em classes sociais e seus diferenciais de condições de saúde. ${ }^{22} \mathrm{O}$ fato de grupos pertencentes a classes sociais escolherem seus locais de residência a partir de condições de renda e acesso a informações não torna a relação entre classe social e lugar biunívoca. Não existem só pobres em um lugar, assim como não existem pobres em um só lugar. Além disso, o fenômeno tratado genericamente como pobreza pode (e deve) ser compreendido como um conjunto de condições extremamente

\section{$O$ espaço pode ser concebido como produtor de diferenciações sociais e epidemiológicas.}


diversificadas, como, por exemplo, a pobreza rural e a urbana, das favelas ou de cinturões metropolitanos. Leon Tostoi ${ }^{23}$ na abertura do livro Ana Karenina declara que "todas as famílias felizes se parecem, mas cada família infeliz o é do seu modo". Cada um desses lugares pode produzir condições de vida e perfis epidemiológicos extremamente diferentes entre si, apesar de contidos dentro de uma mesma categoria social envolvente.

Uma das mais importantes tendências dos estudos sobre desigualdades sociais é o uso de indicadores sintéticos de qualidade de vida, entre eles o Índice de Desenvolvimento Humano (IDH), criado pelo Programa das Nações Unidas para o Desenvolvimento (PNUD). O conceito de "desenvolvimento humano" passou a ser usado em substituição a parâmetros meramente econômicos na medição do desenvolvimento (principalmente o Produto Interno Bruto). Trabalhos, na escala nacional, ${ }^{24}$ regional ${ }^{25} \mathrm{e}$ intramunicipal (por exemplo as Prefeituras de Belo Horizonte e Curitiba) têm sido desenvolvidos, utilizando-se o Índice de Desenvolvimento Humano e outros índices econômicos e sociais para a medição de iniqüidades.

A análise multivariada de um conjunto de indicadores socioeconômicos obtidos dos censos demográficos tem sido uma outra alternativa para a discriminação das regiões que formam este mosaico espacial. ${ }^{26,27}$ Por meio dessas análises, pode-se estabelecer grupos de indicadores que representem "fatores" mais específicos, evitando-se a redundância entre variáveis.

A escolha ou construção de indicadores para medir a relação entre qualidade de vida, ambiente e saúde, envolve importantes aspectos técnicos e políticos. O indicador é um elemento de legitimação dos discursos a respeito de uma determinada realidade que se pretende retratar. A criação e escolha de indicadores carregam implícitos pressupostos e hipóteses do pesquisador. No caso da qualidade de vida e saúde, o indicador ou grupo de indicadores utilizados visam expor a existência de uma desigualdade socioespacial, entendida como uma distribuição desigual de recursos e oportunidades entre os indivíduos e grupos localizados no espaço. Nesse caso, o interesse é mostrar que a diferença de posição relativa no espaço, ou melhor, de localização espacial representa um fator gerador de diferença de acesso aos recursos e oportunidades de vida.

A existência de desigualdades não tem conotação necessariamente negativa. A desigualdade pode ser fruto de um maior esforço de um determinado indivíduo ou grupo de indivíduos que, por isso, pode apresentar num determinado momento melhor condição de vida do que outros. A desigualdade é considerada negativa quando é resultado de uma injustiça, de privilégios ou de ações criminosas. Nesse sentido, a noção de iniqüidade parece ter implícita a distribuição injusta de recursos, o que não acontece com a noção de desigualdade. Por fim, o termo diferença não possui conotação positiva ou negativa. Todos os indivíduos e grupos sociais têm direito de ser diferentes e deve-se, portanto, desenvolver mecanismos que permitam a convivência entre diferentes.

O oposto da igualdade pode significar, tanto a desigualdade, quanto a diferença. Segundo essa última perspectiva, o espaço é formado por lugares marcados pela diferença. Esta abordagem está calcada na ideologia "pós-moderna", no estudo dos modos de vida, em substituição à qualidade de vida. ${ }^{28} \mathrm{O}$ diferente envolve referenciais qualitativos, como aspectos culturais de identidade e representação simbólica. Segundo Raffestin, ${ }^{29}$ a territorialidade de um grupo social é estabelecida pelas relações de alteridade, pela demarcação de limites, além dos quais estão os outros e os objetos que são dos outros. Estes critérios de inclusão e exclusão são subjetivos e coletivos. Os lugares sagrados, campos de atuação de grupos jovens urbanos e guetos são demarcados a partir da diferença em relação ao outro e da identidade do grupo. Nesses casos, não há como estabelecer 
comparações quantitativas entre o que está dentro e o que está fora.

Por outro lado, os estudos sobre desigualdade pressupõem a homogeneidade como paradigma; a adoção de um mesmo referencial, compartilhado por todos, como a renda básica e as condições mínimas de saneamento e de educação. Essas variáveis são contínuas e muitas vezes é esperada uma relação linear entre os indicadores de condição de vida e os indicadores epidemiológicos. Por exemplo, quanto maior a renda de uma população, melhores as condições de saúde, maior a cobertura de sistemas de saneamento e menor a incidência de doenças de veiculação hídrica. Apesar de estas relações serem verificadas freqüentemente por meio de dados empíricos, deve-se estar atento a efeitos não-lineares, de formas de agregação de dados, entre outros. Por exemplo, acima de um determinado nível de renda, as variações de condições de saúde podem ser mínimas.

A diferença pressupõe heterogeneidade. ${ }^{30}$ Para Milton Santos, ${ }^{12}$ a homogeneização é um mito, um "delírio analítico", resultado da difusão da ideologia da globalização. O lugar representa uma resistência a esse processo e procura a afirmação de modos de vida alternativos, em ritmo e valores diferentes do global. Esta identidade coletiva pode, por outro lado, ter conseqüências extremamente negativas para as condições de vida e os ideais de igualdade, como a legitimação do racismo, regionalismo e da intolerância com "o outro". As guerras entre etnias na Europa, Oriente Médio e África são evidências deste processo de afirmação de singularidades e disputa pelo território-nação.

$\mathrm{O}$ uso de indicadores de qualidade de vida e a utilização de técnicas de análise espacial, como o geoprocessamento, são meios de aproximação da realidade e não constituem um fim em si. Fazem parte do processo de investigação de relações possíveis entre organização espacial e saúde. A análise espacial e suas ferramentas devem ser dirigidas ao desafio da solução de problemas con- cretos e baseada em modelos teóricoconceituais apropriados e específicos a cada situação. É importante salientar que a própria apresentação gráfica de resultados em mapas ou diagramas nada tem de objetivo. Um exemplo comum são os gráficos apresentados para ilustrar tendências em uma série histórica. Dependendo das unidades escolhidas para as ordenadas e abscissas, a curva terá inclinação maior ou menor, suavizando ou ressaltando o impacto visual dos dados. No caso dos mapas, o mesmo ocorre. Nesse caso, o recorte espacial e das categorias utilizadas pode alterar a apresentação de tendências, acentuando ou abrandando os aspectos estudados.

\section{Considerações finais}

A análise espacial de condições de saúde pode ser um instrumento importante na avaliação do impacto de processos e estruturas sociais na determinação de eventos de saúde. A categoria espaço tem valor intrínseco na análise das relações entre saúde, sociedade e ambiente. Conhecer a estrutura e a dinâmica espacial da população é o primeiro passo para a caracterização de situações de saúde. Além disso, permite o planejamento de ações de controle e alocação de recursos. A análise espacial propicia o restabelecimento do contexto no qual um evento de saúde ocorre, contribuindo para o entendimento dos processos socioambientais envolvidos.

A análise de situação de saúde é intrinsecamente espacial. Exige a adoção de unidades espaciais para a consolidação de indicadores. Quanto maior a identidade entre a população e seu território, mais facilmente podem ser elaborados os diagnósticos e planejadas as ações de saúde. A situação de saúde não é um atributo dos grupos sociais nem das unidades espaciais em si. Ela é o resultado da relação de grupos sociais com seu território. A perda de vínculos entre população e território, em virtude, por exemplo, da migração, cria uma nova situação de saúde. O processo de desterritorialização e de perda da identidade com

\section{0 indicador ou grupo de indicadores de qualidade de vida $e$ saúde visam expor a existência de uma desigualdade socioespacial.}


o lugar pode ser um importante fator para incorporação e propagação de riscos à saúde coletiva, pela intensificação de fluxos migratórios e desestruturação de redes sociais. ${ }^{31}$

A análise espacial é definida como a capacidade de manipular dados espaciais de diferentes formatos e obter informações adicionais a partir destes. ${ }^{32}$ Esse conceito tem sido utilizado quase unicamente para incentivar o desenvolvimento de técnicas estatísticas espaciais, mas pode ser ampliado para a análise de situações (no sentido etimológico de sítio, lugar) como a posição de um grupo social no espaço influi na maneira como este se relaciona com o ambiente, a sociedade, e, em última análise, suas condições de saúde. Colocar dados no mapa e retirar destes a informação requerida é o sentido do mapeamento em saúde. A forma com que se colocam estes dados no mapa condiciona os possíveis resultados estatísticos e visuais desta análise.

O significado dos diversos conceitos utilizados na abordagem espacial devem ser explicitados, realizando um "controle semântico" para esclarecer o uso e limitações de cada um, tendo em vista as suas origens e incorporações pelas diversas disciplinas envolvidas no trabalho de análise espacial. Nesse caso, é necessário propor novos conceitos que sejam mais aproximados dos estudos empíricos que estão sendo realizados (isto é, ambiente, meio, qualidade de vida, condições de vida, bem-estar, diversidade, desigualdade, diferença, iniqüidade).

As ferramentas de análise citadas devem atingir os diferentes níveis de gestão de serviços (nacional, estadual e municipal), de modo a aumentar a capacidade de análise sobre situação de saúde e ambiente em suas áreas de abrangência, aprimorar o intercâmbio de informações entre seus diversos setores, assim como disponibilizar e divulgar essas informações para a população.

\section{Agradecimentos}

Agradecemos a todos os participantes da oficina de trabalho realizada durante o I Seminário Nacional Saúde e Ambiente no Processo Desenvolimento, entre eles Dina Czeresnia, Gracia Gondim, Gustavo Bretas, Jaime Breilh, Maurício Barreto e Simone Santos, bem como as contribuições resultantes de encontros preparatórios desse seminário: Flavio Nobre, Marília Sá Carvalho, Oswaldo G. Cruz, Trevor Bailey e Wayner Vieira de Souza.

\section{Referências bibliográficas}

1. Teixeira CF, Paim JS, Vilasboas AL. SUS, modelos assistenciais e vigilância da saúde. Informe Epidemiológico do SUS 1998;7(2):7-28.

2. Castellanos PL. Epidemiologia, saúde pública, situação de saúde e condições de vida: considerações conceituais. In: Barradas RB, organizador. Condições de vida e situação de saúde. Rio de Janeiro: Abrasco; 1997. p. 31-76.

3. Carvalho MS, Cruz OG. Análise espacial por micro-áreas: métodos e experiências. In: Veras RP, organizador. Epidemiologia: contextos e pluralidade. Rio de Janeiro: FIOCRUZ; 1998. p.79-89. Série Epidemiológica n. 4.

4. Hills M, Alexander F. Statistical methods used in assessing the risk of disease near a source of possible environmental pollution: a review. Journal of Royal Statistical Society Association 1989;152:353-363.

5. Briggs DJ. Mapping environmental exposure. In: Elliot P. Geographical and environmental epidemiology: methods for small-area studies. Tokyo: Oxford University; 1992. p. 158-176.

6. Bailey TC, Gratell AC. Interactive spatial data analysis. Essex: Longman Scientific \& Technical; 1995.

7. Corrêa RL. Região e organização espacial. São Paulo: Ática; 1986.

8. King PE. Problems of spatial analysis in geographical epidemiology. Social Science \& Medicine 1979;13:249252. 
9. Openshaw S. Fuzzy logic as a new scientific paradigm for doing geography. Environment and Planning A 1996;28(5):761-768.

10. Takahashi LM. Concepts of difference in community health. In: Kearns RA, Gesler WM. Putting health into place: Landscape, identity and wellbeing. Nova York: Syracuse University Press; 1998. p.143-167.

11. Sabroza PC, Leal MC. Saúde, ambiente e desenvolvimento: alguns conceitos fundamentais. In: Leal MC, Sabroza PC, Rodriguez RH, Buss PM, organizadores. Saúde, ambiente e desenvolvimento. v.1. São Paulo: HUCITEC; 1992. p.45-93.

12. Santos M. A natureza do espaço: técnica e tempo, razão e emoção. São Paulo: HUCITEC; 1999.

13. Gesler WM, Bird ST, Oljeski SA. Disease ecology and a reformist alternative: the case of infant mortality. Social Science \& Medicine 1997;44(5):657-671.

14. Norberg E, Finer D. Society, environment and health in lowincome countries. Goteborg, Suécia: Karolinska Institute; 1990.

15. Santos M. Espaço e método. São Paulo: Nobel; 1988.

16. Jolley DJ, Jarman B, Elliot P. Socioeconomic confounding. In: Elliot P. Geographical and environmental epidemiology: methods for small-area studies. Tokyo: Oxford University; 1992. p.115-124.

17. Barcellos C, Santos SM. Colocando dados no mapa: a escolha da unidade espacial de agregação e integração de bases de dados em saúde e ambiente através do geoprocessamento. Informe Epidemiológico do SUS 1997; 6(1):21-29.

18. Vine MF, Degnan D, Hanchette C. Geographic information systems: their use in environmental epidemiologic research. Environmental Health Perspectives 1997;105(6):598-605.

19. Diez-Roux AV. Bringing context back into epidemiology: variables and multilevel analysis. American Journal of Public Health 1998;88:216-222.

20. Carstairs V. Deprivation indices: their interpretation and use in relation to health. Journal of Epidemiology and Community Health 1995;49(2):3-8.

21. Barcellos C, Sabroza PC. Socioenvironmental determinants of the leptospirosis outbreak of 1996 in the western Rio de Janeiro: a geographical approach. International Journal of Environmental Health Research 2000;10:301-313.

22. Duncan BB, Rumel D, Zelmanowicz A, Mengue SS, Santos SM, Dalmáz A. Social inequality in mortality in São Paulo State, Brazil. International Journal of Epidemiology 1995;24(2): 359-365.

23. Tolstoi L. Ana Karenina. Rio de Janeiro: Ediouro; 1996.

24. Programa das Nações Unidas para o Desenvolvimento. Desenvolvimento humano e condições de vida: indicadores brasileiros. Brasília: Programa das Nações Unidas para o Desenvolvimento; 1998.

25. Fundação João Pinheiro. Condições de vida nos municípios de Minas Gerais, 1970-1980-1991. Belo Horizonte: Fundação João Pinheiro; 1996.

26. Paim JS. Abordagens teórico-conceituais em estudos de condições de vida e saúde: notas para reflexão e ação. In: Barata RB. Condições de vida e situação de saúde. Rio de Janeiro: Abrasco; 1997.

27. Carvalho MS, Cruz OG, Nobre FF. Perfil de risco: método multivariado de classificação socioeconômica de microáreas urbanas - os setores censitários da Região Metropolitana do Rio de Janeiro. Cadernos de Saúde Pública 1997;13:435-445.

28. Harvey D. Condição pós-moderna. São Paulo: Loyola; 1993.

29. Raffestin C. Por uma geografia do Poder. São Paulo: Ática; 1993.

30. Haesbaert R. Desterritorialização e identidade: a rede gaúcha no Nordeste. 
Niterói: Universidade Federal Fluminense; 1997.

31. Wallace R. Social desintegration and the spread of Aids. Social Science \& Medicine 1993;38(7):887-896.
32. Bailey TC. A review of statistical spatial analysis in geographical information systems. In: Fotheringham S, Rogerson P. Spatial analysis and GIS. Londres: Taylor \& Francis; 1994. p.13-44. 